\title{
emerald
}

Data Technologies \& Applic

\section{Transfer learning for hostel image classification}

\begin{tabular}{|r|l|}
\hline Journal: & Data Technologies and Applications \\
\hline Manuscript ID & DTA-02-2021-0042.R2 \\
\hline Manuscript Type: & Article \\
\hline Keywords: & $\begin{array}{l}\text { Transfer learning, CNN, Image classification, Hostel image, Artificial } \\
\text { neural network, digital image }\end{array}$ \\
\hline \multicolumn{2}{|l}{} \\
\hline
\end{tabular}

SCHOLARONE ${ }^{\text {m }}$

Manuscripts 
Title

\title{
Transfer learning for hostel image classification
}

\begin{abstract}
Purpose: Due to the fast-growing digital image collections on online platforms and the transfer learning ability of deep learning technology, image classification could be improved and implemented for the hostel domain which has complex clusters of image contents. This paper aims to test the potential of 11 pretrained Convolutional Neural Network (CNN) with transfer learning for hostel image classification on the first hostel image database in order to advance the knowledge and fill the gap academically, as well as to suggest an alternative solution in optimal image classification with less labour cost and human errors to those who manage hostel image collections.

Design/methodology/approach: The hostel image database is first created with data pre-processing steps, data selection and data augmentation. Then, the systematic and comprehensive investigation is divided into seven experiments to test 11 pretrained CNNs which transfer learning was applied and parameters were fine-tuned to match this newly created hostel image dataset. All experiments were conducted in Google Colaboratory environment using Pytorch.
\end{abstract}

Findings: The 7,350-hostel image database is created and labelled into seven classes. Furthermore, its experiment results highlight that DenseNet 121 and DenseNet201 have the greatest potential for hostel image classification as they outperform other CNNs in terms of accuracy and training time.

Originality/value: The fact that no existing academic work dedicating to test pretrained CNNs with transfer learning for hostel image classification and no existing hostel image-only database have made this paper a novel contribution.

Keywords: Transfer learning, hostel image classification, CNN

Article Classification: Research paper

\section{Introduction}


Due to the economic and societal impacts of current coronavirus pandemic on the hostel sector, and other sectors in the global economy, as well as the fast-growing digital image collections on online platforms, it is crucial to utilize the state-of-the-art technology available to organize/classify these images and improve the sector's services/products in order to bounce back to a stronger position and accommodate the changing behaviours of potential customers. Therefore, a brief review of three key areas is addressed as follow.

\subsection{Tourism industry and hostel sector in coronavirus pandemic}

Tourism has been a significant sector of many national economies around the globe and, unfortunately, it has also been one of the most directly affected industry in the current global pandemic of coronavirus (COVID19). According to the Organization for Economic Co-operation and Development (OECD, 2020) report, the COVID-19 impact results to around $80 \%$ decline in international tourism in 2020. Several governments have introduced tourism specific measures to support the industry which some measures are specifically for hostel market. For example, the Belgian government subsidies six million euros to the tourism industry, dedicatedly for the Flemish region with one million euros for youth hostels (Bruegel Datasets, 2020), and the Mayor of London makes 40 million pounds fund available for COVID-19 safe hostels (Greater London Authority, 2020). Despite the economic downturn and tight budget, it can be seen that the governments see the opportunities for economic recovery through the hostel sector. Furthermore, the Hostels Global Market Report 2020-2030 mentions that the market could grow from 6.08 billion US dollars in 2019 to 7.5 billion US dollars in 2023 (Businesswire, 2020).

\subsection{The fast-growing digital image collections}

In spite of a number of major hostel chains such as A\&O, Generator, Meininger, and St. Christopher's Inns, the hostel market consists of countless independent hostels which heavily rely on online travel agencies for their customers as these e-intermediaries play major roles as a fare aggregator and a reservation portal. Some of the key players are www.hostelz.com, providing a list of almost 50,000 hostels in over 7,000 cities, www.hostelworld.com, containing a database of over 36,000 properties, www.hostels.com and www.booking.com, listing over 30,000 hostels on their sites. Furthermore, the fact that the hostel market considers its images as a critical factor as images portrayed its facilities and unique atmosphere which highly influence the decision-making process of potential guests when choosing an accommodation (Bunda, 2014) and the fact that digital image sharing on social media via smartphone has become a phenomenon in today's society, 350 million images are uploaded every day on Facebook, more than 50 billion images so far have been uploaded to Instagram, and over 1.2 billion images and videos are uploaded on Google Photos (Aslam, 2021a; Aslam, 2021b; Sabharwal, 2017; Stadlen, 2019), the vast and growing collections of digital images have become a challenge for those who manage these image data including e-intermediaries' portals such as booking.com where travellers upload more than 150 million images onto the system in order to share their experiences during their stay (Tasli, 2017a). Therefore, to manually or automatically classify this enormous amount of images in their database and systematically organize for future use such as image retrieval or image analysis could be costly and time-consuming, let alone correctly classify these images. In addition, by considering a hostel image, particularly the interior image, it tends to have more complex clusters of image contents when compared to a hotel image. This leads to more difficulty in image classification/organization.

\subsection{Deep learning and transfer learning for image classification}

To organize the huge image collections and fasten the image indexing task, various attempts on image classification have been found in the tourism domain. For example, Amato et al. (2015) conduct a monument recognition experiment using the k-Nearest Neighbour technique, Zheng et al. (2014) apply Scale-Invariant 
However, the accuracy of image classification using CNN highly depends on large scale database as the more information it receives, the more accurate performance results it is likely to provide, high-end computational unit to execute this compute-intensive task in a short period of time which is costly to operate and procure, and the network depth as the deeper network could create more detailed representatives of image input and become more efficient in computation and number of parameters (Shaha and Pawar, 2018a). Therefore, the transfer learning technique is further applied as a solution for image classification task on small scale dataset with less computational cost as its concept is to use a CNN model which is pretrained on a large dataset and transfer some of its learned parameters to the adapted version of the pretrained $\mathrm{CNN}$ in order to perform classification task on a much smaller and different image dataset. Despite the fact that image classification on hostel images is more challenging than hotel images due to more complex clusters of image contents, to the best of our knowledge, there is no academic study on image classification with transfer learning for the hostel sector. Nor an existing database specifically dedicated to the hostel image collection.

As a result, the first hostel image database is created and tested for the potential application of transfer learning on pretrained CNNs for the image classification task. The rest of this paper organized as follows. Section two addresses the relevant literature. Section three provides chosen methodology including the sources and preprocessing steps of the newly created hostel image database, investigated CNN models, and approach for experiments. Results with critical analysis are shown in section four. Lastly, the conclusion and future directions are demonstrated in section five. 


\section{Literature review}

Similar to human judgement, the more information we receive, the better decision we make, it is essential for $\mathrm{CNN}$ to have sufficient information in order to achieve a good result. Therefore, large databases are created to train CNN such as ImageNet which contains over 14 million images and Place365 which contains over 1.8 million images. Several CNN models were developed and extensively trained on these large databases, especially ImageNet which is a large-scale database used in Large-Scale Visual Recognition Challenge, such as AlexNet, Visual Geometry Group (VGG), GoogLeNet, and Residual Network (ResNet) and many of these state-of-the-art neural networks are widely used in image classification tasks. For example, Dawud et al. (2019a) and Zhao et al. (2017a) applied AlexNet in their experiment, Li et al. (2020a), Mateen et al. (2020a), Shaha and Pawar (2018b) and Stylianou et al. (2019b) used VGG, Szegedy et al. (2015) preferred GoogLeNet, and Mateen et al. (2020b), Stylianou et al. (2019c), and Xiao et al. (2018a) chose ResNet.

However, for image classification task or other tasks on a much smaller database, even with data augmentation applied by executing rotation, horizontal flipping, vertical flipping, or resizing images in the database in order to provide more information to CNN model via these artificial increase of training images, it could be difficult for the model-CNN to learn millions of its network parameters and provide good performance as it is likely to overfit the training data with low bias which lead to false performance. As a result, transfer learning technique is introduced to small scale database tasks as an alternative to deep learning the $\mathrm{CNN}$ from scratch. The process of transfer learning can be explained in two steps, importing the fully pretrained CNN construction and finetuning its parameters, as illustrated in figure 2 below.

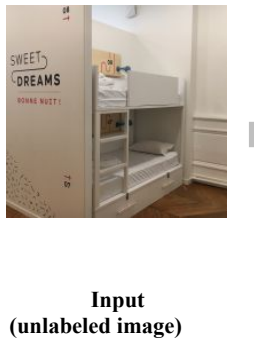

(unlabeled image)
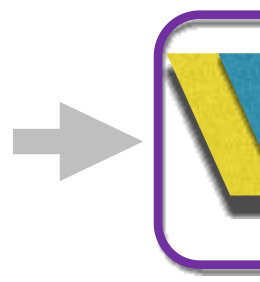

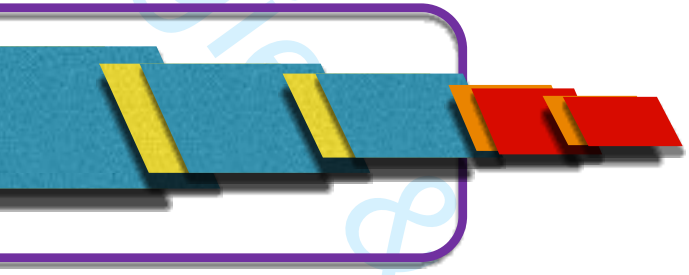

Transferred deep layers (pretrained CNN)
New deep layers (finetuned parameters)

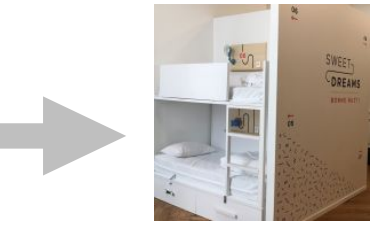

Output (classified image: bunk bedroom)

Figure 2: CNN transfer learning for image classification

To reduce computational cost and achieve high accuracy, transfer learning takes advantage of pretrained CNN by using the learned parameters/weights from its earlier layers which extract low-level features such as edges, patterns, corners, and gradients which are general features in any image. Despite different database used on the pretrained CNN, these low-level features are similar and can be transferred to the new task resulting in increasing the feature extraction capability of the adapted CNN by requiringwith less computational time in the training processeost. The nNext step is to fine-tune parameters by making small adjustments on parameters/weights of the pretrained CNN on the latter layers which extract high-level features such as objects and events which are specific features in a given image in order to match new dataset and then, add a new classifier and train this adapted CNN for the new task. This fine-tuning step can be repeated until the desired result is achieved. Transfer learning does not only help to fasten the overall process of image classification but also reduces computational resources used such as large storage space, high-speed network connection, and multi-core processors. Furthermore, better performance could be achieved by using a lot fewer data during the training of adapted CNN. Despite different database used on the pretrained CNN, the low tevel features are

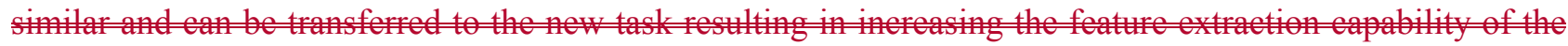
ata CNA a 
Several previous works apply transfer learning by directly employing the pretrained CNN and fine-tuning it for their databases such as Shaha and Pawar (2018c), Stylianou et al. (2019d), Xiao et al. (2018b) and Zhao et al. (2017b) and some experiments additionally modify the pretrained CNN by changing its layers (Dawud et al.,2019b; Li et al., 2020b; Mateen et al, 2020c; Zhao et al., 2017c). Moreover, there is evidence of transfer learning application on CNN features on hotel images such as hotel recognition for human trafficking investigation (Stylianou et al., 2019a), hotel image classification using deep learning (Kanchinadam, 2016), hotel photo content analysis (Ren et al., 2020), Kayak's hotel image categorization with deep learning (Rath, 2018), and automated hotel image tagging (Tasli, 2017b). Furthermore, Kaggle (2016) launched a competition on hotel image classification to encourage students applying deep learning to a real-world problem. Nonetheless, there is no relevant evidence on hostel image experiment and it is not necessary that the high accuracy of the same pretrained CNN which used ImageNet database is transferable to other tasks which use different datasets (MathWorks, 2021). As a result, the hypothesis of this study is constructed below.

Hypothesis: Transfer learning of pretrained CNNs provides good performance in hostel image classification

\section{Research methodology}

As the methodology is a system of ways used to conduct research which is linked to ontology and epistemology, two different types of research, primary research and secondary research, are described by Mason (2014), according to its source of information. Therefore, this study is considered primary research as the testing potential of 11 pretrained CNNs with transfer learning for hostel image classification has not been investigated before. Additionally, modelling/simulation method is chosen to test the algorithms resulting in quantitively outcomes.

\subsection{Hostel image database}

\section{A. Data sources}

According to the relevant literature, the majority of relevant experiments used an existing database(s) (Abdullahzadeh and Mohanna, 2013; Wengert et al., 2011; Zhu et al.,2015b), whereas some experiments used images specifically photographed for their research projects (Premchaiswad et al.,2010; Raisi et al., 2011), and some experiments combined both data collection methods to create their image database (Raisi et al., 2014). Because of this, data collection methods are subjective. Therefore, in the case of this experiment, hostel images are collected from five freely available sources which are online travel agencies 'websites such as www.booking.com and www.hostelworld.com, Google, Flickr, Places365 database, and by taking photographs at the premises, where possible, to create the first hostel image-only database which has hostel images taken by both professional and non-professional photographers. Consequently, as various data sources are included, images in this database could represent different hostel designs which varying from traditional to modern, simple to eclectic, and/or budget to boutique. Moreover, images of hostels across the world are considered in order to reduce bias. Lastly, a variety of image quality from the professional and the nonprofessional and time that these images are available and collected, as of December 2020, could be welldemonstrated the real-world digital image collections on current online platforms. 


\section{$\underline{\text { Data selection }}$}

Similar to the data collection method, it seems there is no academic scholar suggests a number of reliable data size or how to calculate the appropriate data size for research in relation to image classification. Nonetheless, the key advantage of transfer learning is to improve classification accuracy when using deep features on small scale datasets and it is evidenced in Rath's experiment (2020a) that for the 8,677-image dataset the classification performance can reach $95 \%$ accuracy. Therefore, in this work, $\mathbf{7 , 3 5 0}$ hostel images are collected which consist of images from chain hostels, hostels which are corporately owned and usually have a similar design, and independent hostels, hostels which are usually privately owned and have unique designs. These images are chosen from hostels in different locations such as the United States in North America, the United Kingdom in Europe, Thailand in Asia, and Ghana in Africa. Moreover, oOnly interior images which contain complex clusters are considered in order to demonstrate the greater challenge of hostel images when compared to hotel images as it is more likely that there are more than two beds in the same room in a hostel bedroom, there are more than one washing machine/dryer in a hostel laundry room, there are more than one shower/toilet in a hostel bathroom, and there are more objects in a hostel kitchen/living room.- Therefore, it is possible that hostel image features consist of vaster feature types and levels such as many colours, many textures, and many shapes which account for the higher number of features to consider when performing classification task. Then, tThe dataset is divided into two sets, training/validation set and test set. The training/validation set consists of the training set which is used to train the algorithm/model and the validation set which is used to unbiasedly evaluate the algorithm/model while fine-tuning parameters, containing 7,000 images. The test set, containing 350 images, is used to unbiasedly evaluate the final algorithm/model. Images in training/validation set are labelled and grouped into seven classes, normal bedroom, bunk bedroom, other bedroom, bathroom, kitchen, living room, and laundry room. Since the bedroom is the key feature for hostel accommodation, three out of seven classes are allocated to three types of bedroom. Furthermore, each class contains $\mathbf{1 , 0 0 0}$ images equally in the training/validation set and $\mathbf{5 0}$ images equally in the test set in order to control this variable which might affect the test performance in this study. Additionally, despite these images are in different sizes, all of them $\underline{\text { are, }}$ in three colour channels and stored in JPEG format as suggested by Wallace et al. (1991) as they illustrated that JPEG is an appropriate still image format for the use of image retrieval /classification purpose. For noncommercial research and educational purposes, this dataset is made available on Google Drive (2021) and hostel image examples of each class are demonstrated in figure 3 below.

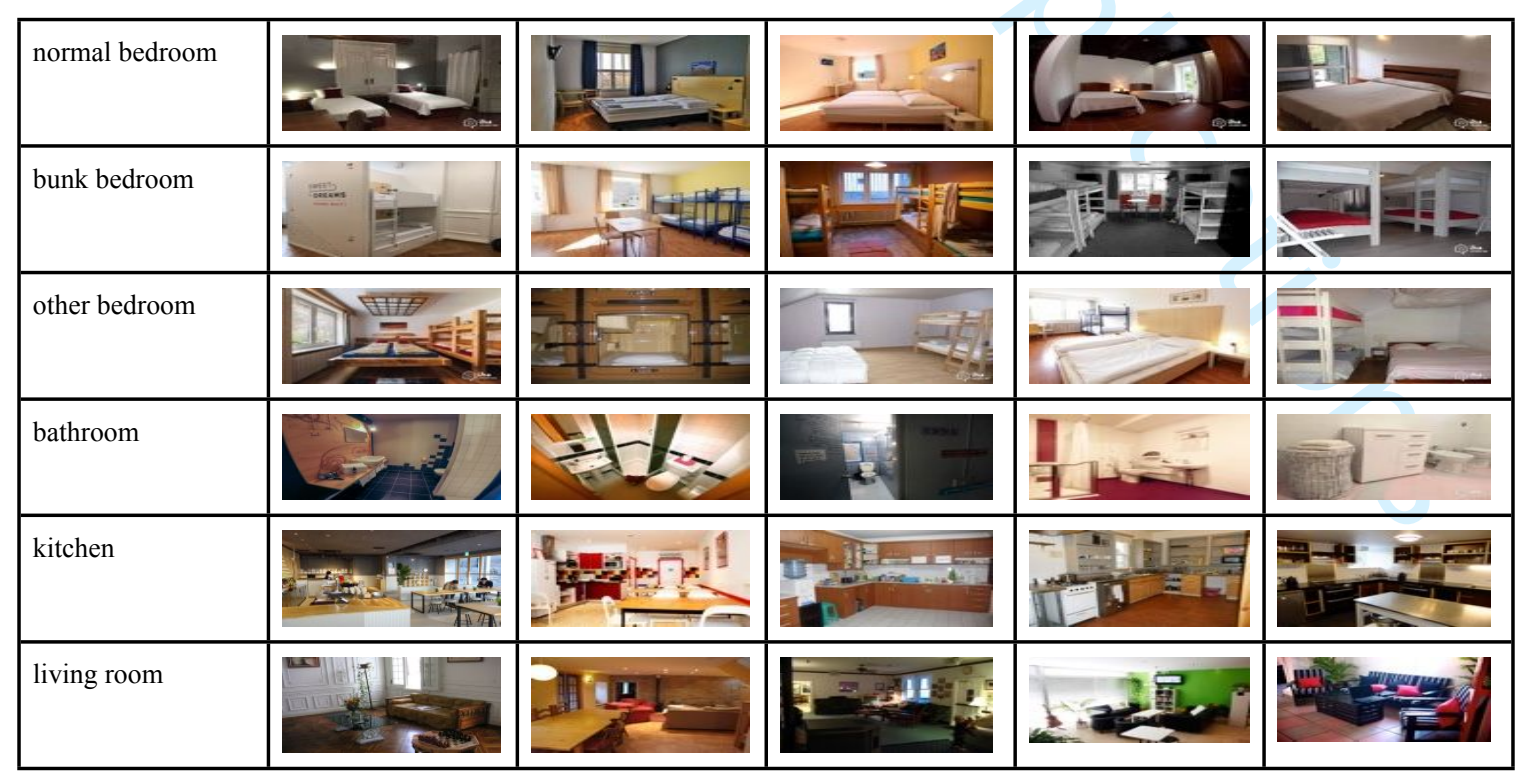




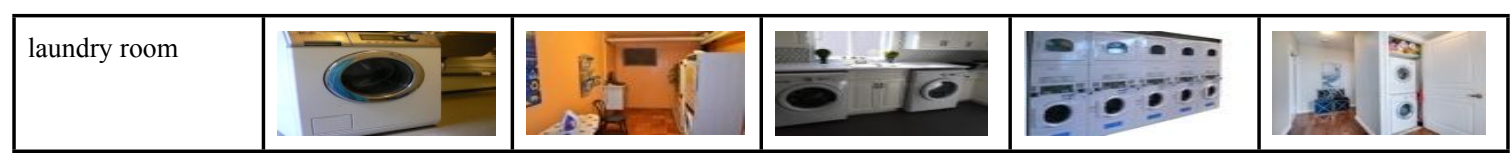

Figure 3: Hostel image examples of each class

\section{$\underline{\text { Data augmentation }}$}

In order to help increasing accuracy of this deep learning experiment, data augmentation is applied to provide multiplied data to the system which could also help to prevent the overfitting during training. Image data can be augmented by rotation, horizontal flipping, vertical flipping, shifting, and/or resizing.

Furthermore, some of the regularization techniques are also applied in order to facilitate CNN models. These techniques include weight sharing, dropout, early stopping, batch normalization, noise injection, and parameter norm penalties.

\subsection{Pretrained CNN models}

Although more complex models might lead to more accurate results, to be compatible with digital image sharing on mobile devices, in this paper 11 small-size CNN models are explored which are SqueezeNet models (SqueezeNet1_0 and SqueezeNet1_1), ResNet models (ResNet18, ResNet34, ResNet50, ResNet101, and ResNet152), and DenseNet models (DenseNet121, DenseNet161, DenseNet169, and DenseNet201). SqueeneNet models are the most compact networks available which take advantage of fire module using only $1 \times 1$ filters in squeezed convolutional layers and a mix of $1 \times 1$ and $3 \times 3$ filters in expanded convolutional layers. ResNet models provide optimized solutions to the degradation problem, which tends to cause by an increase of deep layers, by letting the stacked layers fit a residual mapping instead of the underlying mapping. Similar to ResNet models, the DenseNet models add shortcuts in deep layers but also concatenate the outputs from previous layers in deep dimensions. Therefore, DenseNet models use fewer parameters than ResNet to represent image features. Moreover, models with different numbers of deep layers are included for a comprehensive approach and their accuracies and relative prediction time using GPU of these models are also feasible for this study. The information about the chosen CNN models is demonstrated as follow.

\begin{tabular}{|l|c|c|c|}
\hline \multicolumn{1}{|c|}{ CNN model } & Model size & No. of deep layer & No. of parameter \\
\hline $\begin{array}{l}\text { SqueezeNet1_0 } \\
\text { (Iandola } \text { et al., 2017) }\end{array}$ & $5.2 \mathrm{MB}$ & 18 & 1.2 million \\
\hline $\begin{array}{l}\text { SqueezeNet1_1 } \\
\text { (Iandola } \text { et al., 2016) }\end{array}$ & $5.2 \mathrm{MB}$ & 18 & 1.2 million \\
\hline $\begin{array}{l}\text { ResNet18 } \\
\text { (He } \text { et al., 2015a) }\end{array}$ & $44 \mathrm{MB}$ & 18 & 11.7 million \\
\hline $\begin{array}{l}\text { ResNet34 } \\
\text { (He } \text { et al., 2015b) }\end{array}$ & $87 \mathrm{MB}$ & 34 & 21.2 million \\
\hline $\begin{array}{l}\text { ResNet50 } \\
\text { (He } \text { et al., 2015c) }\end{array}$ & $96 \mathrm{MB}$ & 50 & 25.6 million \\
\hline
\end{tabular}




\begin{tabular}{|l|c|c|c|}
\hline $\begin{array}{l}\text { ResNet101 } \\
(\text { He } \text { et al., 2015d) }\end{array}$ & $167 \mathrm{MB}$ & 101 & 44.6 million \\
\hline $\begin{array}{l}\text { ResNet152 } \\
(\text { He } \text { et al., 2015e) }\end{array}$ & $230 \mathrm{MB}$ & 152 & 58.2 million \\
\hline $\begin{array}{l}\text { DenseNet121 } \\
\text { (Huang } \text { et al., 2018a) }\end{array}$ & $33 \mathrm{MB}$ & 121 & 7.2 million \\
\hline $\begin{array}{l}\text { DenseNet161 } \\
\text { (Huang } \text { et al., 2018b) }\end{array}$ & $107 \mathrm{MB}$ & 161 & 12.3 million \\
\hline $\begin{array}{l}\text { DenseNet169 } \\
\text { (Huang } \text { et al., 2018c) }\end{array}$ & 57MB & 169 & 20 million \\
\hline $\begin{array}{l}\text { DenseNet201 } \\
\text { (Huang } \text { et al., 2018d) }\end{array}$ & $77 \mathrm{MB}$ & 201 & \\
\hline
\end{tabular}

Table I: CNN models information

\subsection{Experiments}

This study is conducted in the Google Colaboratory environment, a free cloud service with free graphics processing unit (GPU), using Pytorch on Intel(R) Core (TM) i7-6700 CPU @3.40GHz machine with 16GB RAM and NVIDIA Tesla K80 GPU with 12GB memory. The implemented Pytorch code is an adapted version of Rath's algorithm (2020b). The approach is to directly employ 11 pretrained CNNs. Then, transfer learning is implemented with fine-tuning some parameters on the latter layers to match the hostel image database collected. Furthermore, all images, 7,000 images, in training/validation set are randomly rotated between -30 and 30 degrees, centre cropped and resized to $224 \times 224$ format as an input format required by the selected pretrained CNNs, and horizontally flipped for data augmentation process. On top of this, batch normalization which is a supervised learning technique that standardizes the inputs in a neural network layer, weight sharing which is a technique that uses the same numbers within each neuron/filter in a particular layer, and dropout which is a technique that ignores randomly selected neurons during training are chosen as the regularization techniques to facilitate these $\mathrm{CNN}$ models.

SqueezeNet1_0 model is selected as a CNN baseline in this study which is divided into seven experiments as follow:

1st experiment is to test the baseline performance for five epochs on various proportions of data split in training/validation set (90:10, 80:20, 70:30, 60:40, and 50:50) and its effect on the test set performance;

2nd experiment is to test the baseline performance for five epochs on various dropout rate in training/validation set $(0.1,0.2,0.3,0.4$, and 0.5$)$ and its effect on the test set performance;

3rd experiment is to test 11 pretrained CNNs performances at the best-performed data split and dropout rate;

4th experiment is to test 11 pretrained CNNs performance for various epochs at the best-result data split and dropout rate;

5th experiment is to test 11 pretrained CNNs performance for 25 epochs at the best-result data split and dropout rate and its effect on the test set performance; 


\subsection{Performance measurement}

To evaluate the transfer learning performance of pretrained CNN models on hostel image classification task, two measures are implemented as follow.

A. Accuracy is a ratio of the number of correctly classified images to the total number of all images.

Accuracy $=\frac{\text { The number of correctly classified images }}{\text { The total number of all images }}$

B. Training time (minutes)

\section{Results with critical analysis}

\subsection{1st experiment}

\begin{tabular}{|c|c|c|}
\hline \multirow{2}{*}{$\begin{array}{c}\text { Data split } \\
\text { (Training set: Validation set) }\end{array}$} & \multicolumn{2}{|c|}{ Accuracy (\%) } \\
\cline { 2 - 3 } & Validation set & Test set \\
\hline $90: 10$ & $\mathbf{7 4 . 2 2 8}$ & 70.857 \\
\hline $80: 20$ & 72.626 & 69.714 \\
\hline $70: 30$ & 72.960 & 70.286 \\
\hline $60: 40$ & 69.472 & 67.143 \\
\hline $50: 50$ & 66.924 & $\mathbf{7 1 . 7 1 4}$ \\
\hline
\end{tabular}

Table II: An accuracy comparison of SqueezeNet1_0 on validation set \& test set at different data split. Bold values indicate the highest accuracy and the optimal option is highlighted.

Looking at an accuracy of SqueezeNet1_0 (baseline CNN) on the validation set, it can be seen that the data split between the training set and the validation set at 90:10 provides the highest accuracy, $74.228 \%$, whereas the accuracy on test set demonstrates the highest at 50:50 proportion, $71.714 \%$. Having said this, as one proportion can only be selected to apply in further experiments, the accuracies on validation set and test set need to be considered together. Despite the data split at 90:10 achieves the best results, the 70:30 proportion could also be considered as it provides the second-best results. Therefore, due to the benefit of validation set which is to provide an unbiased performance of the training model, in the 2nd experiment data split at 90:10 
and 70:30 are tested to finalize the most appropriate data proportionis for the rest of the experiments. Additionally, it is worth noting that even though a low-cost model like SqueezeNet1_0 is implemented, the classification performances are reasonable for mobile application.

\subsection{2nd experiment}

\begin{tabular}{|c|c|c|c|c|}
\hline \multirow{2}{*}{ Dropout } & \multicolumn{3}{|c|}{} & \multicolumn{2}{|c|}{ Accuracy (\%) } \\
\cline { 2 - 5 } & & $\underline{2}$ & Validation set & Test set \\
\hline 0.1 & $\underline{71.769}$ & $\underline{69.376}$ & 71.632 & 70.000 \\
\hline 0.2 & $\underline{67.395}$ & $\underline{61.958}$ & 68.966 & 62.571 \\
\hline 0.3 & $\underline{\mathbf{7 2 . 1 4 0}}$ & $\underline{69.965}$ & $\mathbf{7 2 . 9 6 0}$ & 70.286 \\
\hline 0.4 & $\underline{69.264}$ & $\underline{70.011}$ & 69.748 & 70.571 \\
\hline 0.5 & $\underline{64.752}$ & $\underline{\mathbf{7 1 . 3 7 8}}$ & 63.378 & $\mathbf{7 2 . 5 7 1}$ \\
\hline
\end{tabular}

Table III: An accuracy comparison of SqueezeNet1_0 on validation set \& test set at different dropout at 90:10 and 70:30 data split. Bold values indicate the highest accuracy and the optimal option is highlighted.

Considering an accuracy of the baseline $\mathrm{CNN}$ on the validation set, at 0.3 dropout rate results in the highest accuracy, $72.140 \%$ at $90: 10$ data split and $72.96 \%$ at $70: 30$ data split, whereas on the test set at 0.5 dropout achieves the highest result, $71.378 \%$ at $90: 10$ data split and $72.571 \%$ at $70: 30$ data split. Similar to the 1 st experiment on different data splits, it is essential to consider the accuracies on the validation set and test set all together at one dropout rate. Consequently, as shown in table III, the dropout rate at 0.3 contributes to the highest accuracies of SqueezeNet1_0 after transfer learning and therefore, the remaining experiments also apply the 0.3 dropout rate. As for the most appropriate data proportion, despite 90:10 data split provides the highest accuracy in the 1st experiment, when considering different dropout 70:30 data split performs better in many cases. Therefore, for the rest of the experiments, 70:30 proportion is applied.

\subsection{3 rd experiment}

\begin{tabular}{|c|c|c|}
\hline CNN model & Accuracy (\%) on validation set & Training time (mins) \\
\hline SqueezeNet1_0 & 80.501 & 18.021 \\
\hline SqueezeNet1_1 & 79.827 & $\mathbf{1 7 . 0 0 5}$ \\
\hline ResNet18 & 87.164 & 17.672 \\
\hline ResNet34 & 87.725 & 20.593 \\
\hline ResNet50 & 87.640 & 28.181 \\
\hline ResNet101 & 87.718 & 39.399 \\
\hline ResNet152 & 87.576 & 50.891 \\
\hline DenseNet121 & 89.391 & 35.951 \\
\hline DenseNet161 & 89.557 & 53.737 \\
\hline DenseNet169 & 89.443 & 44.912 \\
\hline DenseNet201 & $\mathbf{8 9 . 7 2 2}$ & 51.691 \\
\hline
\end{tabular}


Table IV: An accuracy and training time comparison of 11 pretrained CNNs after transfer learning. Bold values indicate the best

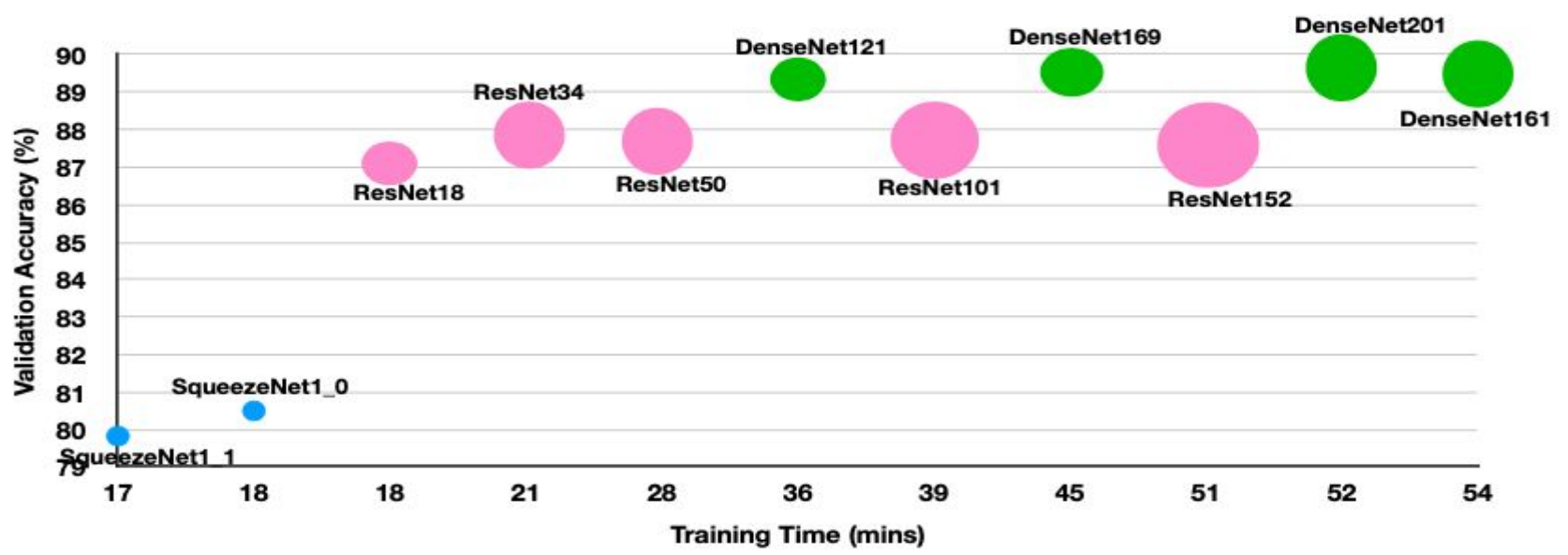

performance and the optimal options are highlighted.

Figure 4: An accuracy and training time comparison of 11 pretrained CNNs after transfer learning

As demonstrated in figure 4, it can be seen that DenseNet models, especially DenseNet201, outperform the other CNN models after applying the transfer learning technique. Nonetheless, if considering only training time, SqueezeNet models spend less computational time due to their smaller model sizes which are illustrated in smaller circles. Having said this, when taking both accuracy and training time into account, it seems that DenseNet121 is one of the most potential models for hostel image database. Therefore, in further experiments, DenseNet121 and DenseNet201 are in focus.

\subsection{4th experiment}

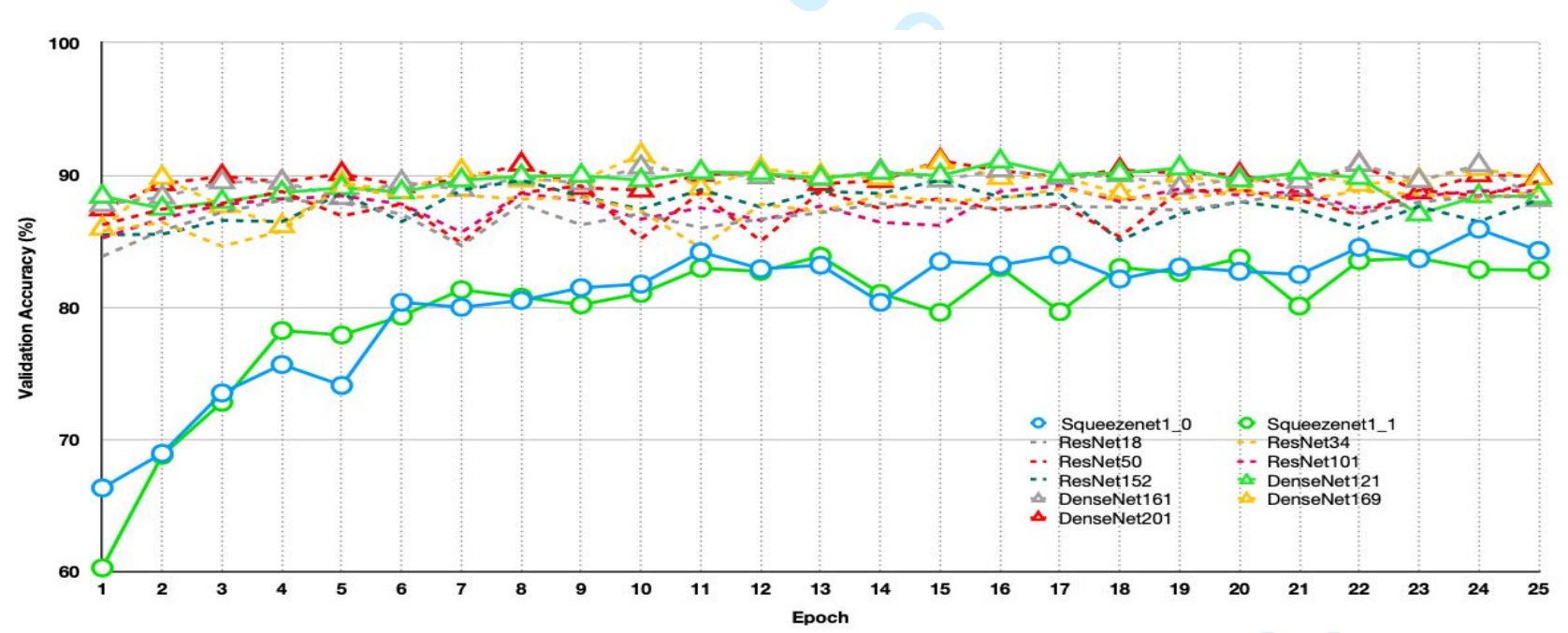

Figure 5: An accuracy comparison of 11 pretrained CNNs after transfer learning at different epoch

When testing pretrained CNNs at different epoch, figure 5 shows that DenseNet models and ResNet models provide similar and stable performance across each of these models, whereas SqueezeNet models start to perform better at six epochs. After this, their accuracy improves slightly and become more stable. Therefore, it can be seen that the CNN models could achieve high accuracies with only a few iterations. Having said this, in order to continue investigating SqueezeNet models at their best performances, including SqueezeNet1_0 as a baseline $\mathrm{CNN}$, the rest of the experiments are conducted at 25 epochs.

\subsection{5th experiment}




\begin{tabular}{|c|c|c|}
\hline \multirow{2}{*}{ CNN model } & \multicolumn{2}{|c|}{ Accuracy (\%) } \\
\cline { 2 - 3 } & Validation set & Test set \\
\hline SqueezeNet1_0 & 80.501 & 81.429 \\
\hline SqueezeNet1_1 & 79.827 & 82.000 \\
\hline ResNet18 & 87.164 & $\mathbf{8 8 . 5 7 1}$ \\
\hline ResNet34 & 87.725 & 88.286 \\
\hline ResNet50 & 87.540 & 83.429 \\
\hline ResNet101 & 87.718 & 86.857 \\
\hline ResNet152 & 87.576 & 83.143 \\
\hline DenseNet121 & 89.391 & 83.714 \\
\hline DenseNet161 & 89.557 & 83.429 \\
\hline DenseNet169 & 89.433 & 85.714 \\
\hline DenseNet201 & $\mathbf{8 9 . 7 2 2}$ & 86.857 \\
\hline
\end{tabular}

Table V: An accuracy comparison of 11 pretrained CNNs after transfer learning on validation set \& test set. Bold values indicate the highest accuracy and the optimal options are highlighted.

It can be seen in table $\mathrm{V}$ that in most CNNs simulations the accuracies on validation set are higher than the accuracies on the test set. However, four models, SqueezeNet1_0, SqueezeNet1_1, ResNet18, and ResNet34, have the opposite results. It could be assumed that the lesser deep layers, the more it becomes overfitting. Nonetheless, the accuracies of DenseNet121 and DenseNet201 on both validation set and test set are still considerably high, $89.391 \%$ and $83.714 \%$ for DenseNet 121 and $89.722 \%$ and $86.857 \%$ for DenseNet201, which reassure their potential for hostel image classification with transfer learning.

\subsection{6th experiment}

\begin{tabular}{|c|c|c|c|c|c|c|}
\hline \multirow{3}{*}{ CNN model } & \multicolumn{3}{|c|}{ Original size database } & \multicolumn{3}{|c|}{ Resized database } \\
\hline & \multicolumn{2}{|c|}{ Accuracy (\%) } & \multirow{2}{*}{$\begin{array}{l}\text { Training time } \\
\text { (mins) }\end{array}$} & \multicolumn{2}{|c|}{ Accuracy (\%) } & \multirow{2}{*}{$\begin{array}{l}\text { Training time } \\
\text { (mins) }\end{array}$} \\
\hline & Validation set & Test set & & Validation set & Test set & \\
\hline SqueezeNet1_0 & 76.260 & 81.429 & 7.208 & 71.200 & 77.143 & 3.999 \\
\hline SqueezeNet1_1 & 76.059 & 82.000 & 6.802 & 75.353 & 77.143 & 3.726 \\
\hline ResNet18 & 86.564 & 88.571 & 7.069 & 87.173 & 86.571 & 4.007 \\
\hline ResNet34 & 87.237 & 88.286 & 8.237 & 87.728 & 87.143 & 4.858 \\
\hline ResNet50 & 87.284 & 83.429 & 11.272 & 87.338 & 86.857 & 8.209 \\
\hline ResNet101 & 87.307 & 86.857 & 15.760 & 87.071 & 81.143 & 12.118 \\
\hline ResNet152 & 87.337 & 83.143 & 20.356 & 87.484 & 84.857 & 16.140 \\
\hline DenseNet121 & 88.932 & 83.714 & 14.380 & 88.557 & 88.571 & 9.954 \\
\hline DenseNet161 & 89.145 & 83.429 & 21.495 & 90.048 & 87.429 & 17.143 \\
\hline DenseNet169 & 88.876 & 85.714 & 17.965 & 89.419 & 85.714 & 12.505 \\
\hline DenseNet201 & 89.376 & 86.857 & 20.676 & 89.296 & 86.286 & 15.180 \\
\hline
\end{tabular}

Table VI: An accuracy comparison of 11 pretrained CNNs on original size database \& resized database at 10 epochs. Bold values indicate the best performance and the optimal options are highlighted.

Due to different image data sources, gathered hostel images come in various image sizes. This 6th experiment tests another hostel image database, which is the same database in the previous experiments but reduced its image sizes to one image size $(300 \times 300)$ prior and in addition to data augmentation in data pre-processing 
stage. Table VI shows that accuracies on validation set are not affected much by resizing the database and remain similar in most CNN cases. Having said this, accuracies on the test set improve slightly in some CNN models, including DenseNet121 which is increased from $83.714 \%$ to $88.571 \%$. Furthermore, the computational time is significantly decreased on the resized database for all CNN models.

\subsection{7 th experiment}

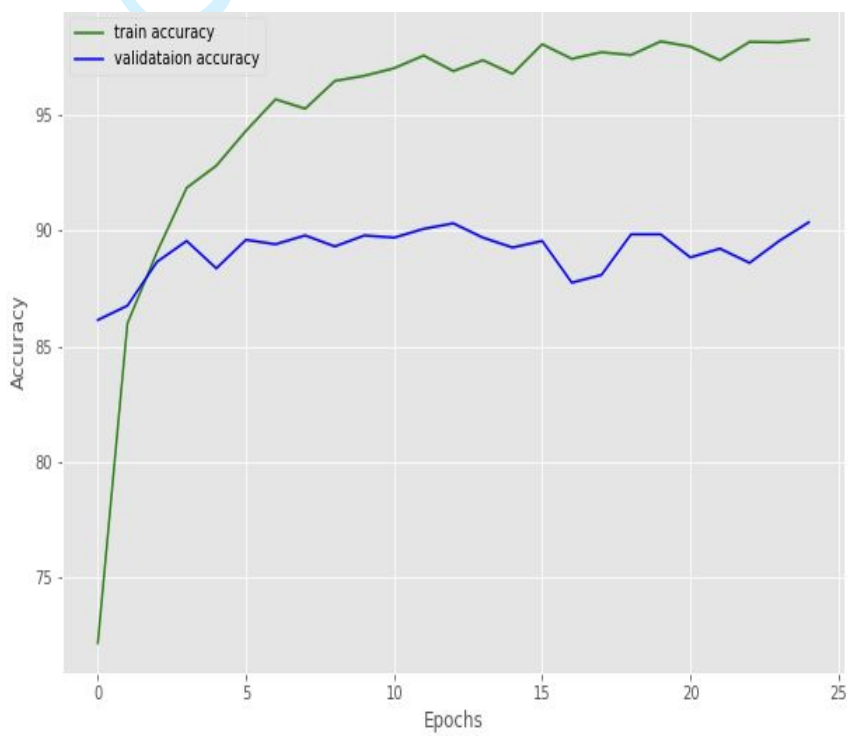

DenseNet121

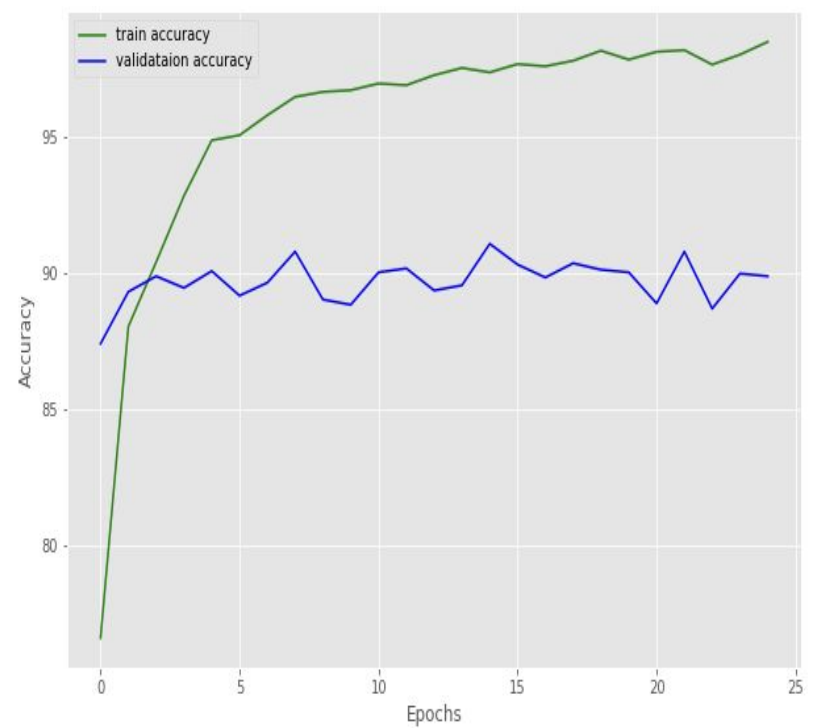

DenseNet201

Figure 6: An accuracy comparison of DenseNet121 \& DenseNet201 on training set \& validation set

Figure 6 illustrates the plots of the accuracy of DenseNet121 and DenseNet201 on both the training set, green colour, and validation set, blue colour. It is clear that validation accuracies remain stable at around $90 \%$ for both models. That means the models perform well and are not overfitting on the current database size. Therefore, the database size could be increased in order to potentially increase the accuracy of hostel image classification.

\section{Conclusion and future directions}

\subsection{Conclusion}

In response to the fast-growing digital image collections on online platforms and the emerging transfer learning technology for image classification, as well as the impacts of coronavirus pandemic on hostel market, this study tested the potential of 11 pretrained CNNs with transfer learning technique for hostel image classification on the first hostel image database which is created for this project. The results from seven experiments highlight that, with the optimization of parameter fine-tuning, various pretrained CNNs with transfer learning can provide good performance. Particularly, DenseNet121 and DenseNet201 outperform the other CNNs, in terms of accuracy rate and training time, and achieve impressive outcomes for hostel image classification. Different elements have been explored in order to find the optimal combination and achieve the highest classification performance. SqueezeNet1_0 is selected as a baseline CNN due to its most compact model size, the smallest number of deep layer, and less number of parameter. In terms of the data split during model training, it shows that the training set and the validation set should be split into $70 \%$ and $30 \%$, respectively. The dropout rate at 0.3 contributes to the highest accuracy during training and relatively high during testing. 
When all 11 pretrained CNNs are considered, the classification accuracy can reach its peak with only a few iterations and become stable for DenseNet models and ResNet models, whereas SqueezeNet models reach $80 \%$ accuracy at six epochs and continue to improve slightly. DenseNet201 best-performs during training and relatively similar during testing, while DenseNet121 demonstrates competitive results. Furthermore, when the size of the hostel image database is reduced, in addition to the data augmentation, the computational time is significantly decreased, even though the classification accuracies only slightly increase. Based on these results, it can be seen that, by applying this state-of-the-art transfer learning technique in pretrained CNNs for image classification, hostel image managers could automatically organize their growing collections and fasten the image indexing task with high accuracy, less labour cost, and less human errors on this accommodation type which has complex clutters of content on an image. Additionally, this optimal solution could contribute to the improved services/products of the hostel sector by utilizing the correctly classified images in database and offering more targeted solutions to their current or potential customers which could lead to a better position to recover from the current global economic downturn.

\subsection{Future directions}

Despite the promising results from this study, it is undeniable that there is plenty of room to further investigate. Suggestions for future directions are stated as follow.

\section{A. CNN layer modification}

Due to the operation of the latter layers of pretrained CNNs which is to extract high-level image features, in addition to fine-tuning parameters to match new image classification task, further modification on some of these layers could be explored in order to compare its results with the fine-tuned only version of the pretrained CNNs. Consequently, the classification accuracy could be improved, as well as the computational cost.

\section{B. PlacesCNN models comparison}

Even though available pretrained CNN models were originally trained on ImageNet database which contains object-centric image data, Zhou et al. (2017) gathered Places365 database, which contains a large-scale scenecentric image data, and trained various CNNs from scratch with this database without changing network architectures. Therefore, by comparing the results from pretrained Places365-CNNs with the original pretrained CNNs could benefit future experiments on hostel image classification task.

\section{Building small size $\mathrm{CNN}$ model}

Since smartphone users are expected to increase from 2.5 billion in 2016 to 3.8 billion in 2021 (O'Dea, 2021日), small size CNN model with good performance like SqueezeNet models could be created in order to facilitate mobile applications on image classification task and other computer vision tasks in the future.

D. Video classification

Apart from a vast collection of digital images on online platforms, it is undeniable that video is another data type that is enormously created in today's society. TankovskaClement (2021日) reports that, as of May 2019, more than 500 hours of video were uploaded to YouTube in every minute and this number of video content 
hours grew by around $40 \%$ between 2014 and 2019. Therefore, video classification could be further researched to accommodate this continuously increasing demand.

\section{E. Image retrieval application}

As the image classification process is a part of image retrieval task, it is crucial to progress to the image retrieval step in order to gain greater benefits. In particular for hostel search by image, can also be called content-based image retrieval, the hostel image managers could practically take advantage of the results from this study and further apply the state-of-the-art knowledge and technology to their real-life business operation.

\section{References}

Abdullahzadeh, A. and Mohanna, F. (2013), "Content-based image retrieval based on affine noisy invariant colour region", International research journal of applied and basic sciences, Vol. 6 No. 5, pp.598-606.

Amato, G., Falchi, F. and Gennaro, C. (2015), "Fast image classification for monument recognition”, available at: https://dl.acm.org/doi/pdf/10.1145/2724727 (accessed $\underline{3}+\underline{\text { JuneMay 20210) }}$

Aslam, S. (2021a), "Facebook by the numbers: stats, demographics \& fun facts", available at: https://www.omnicoreagency.com/facebook-statistics/ (accessed $\underline{3}+\underline{\text { JuneFebruary 2021) }}$

Aslam, S. (2021b), "Instagram by the numbers: stats, demographics \& fun facts", available at: https://www.omnicoreagency.com/instagram-statistics/ (accessed 31 JuneFebruary 2021)

Bruegel Datasets (2020), "The fiscal response to the economic fallout from the coronavirus", available at: https://www.bruegel.org/publications/datasets/covid-national-dataset/\#belgium (accessed 31 Juneanury 2021)

Bunda, R. (2014), "The business of beds: an exploration hotel and hostel business strategy", available at: https://opencommons.uconn.edu/cgi/viewcontent.cgi?article=1358\&context=srhonors_theses (accessed $\underline{3} 1 \underline{\text { JuneMay 2021 }} 18$ ) Businesswire (2020), "Impact of COVID-19 on the global hostels market 2020-2030", available at: https://www.businesswire.com/news/home/20200615005443/en/Impact-of-COVID-19-on-the-Global-Hostels-Market-20202030---ResearchAndMarkets.com (accessed $\underline{3}+$ Juneanuary 2021)

Clement, J. (2020) "Hours of vide uploaded to YouTube every minute 2007 2019", available at:

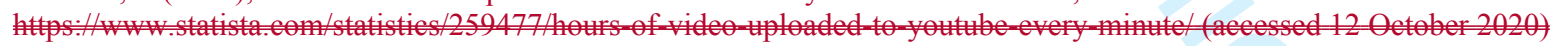

Dawud, A., Yurtkan, K. and Oztoprak, H. (2019), “Application of deep learning in neuroradiology: brain haemorrhage classification using transfer learning “, Computational intelligence and neuroscience, Vol. 2019, pp.1-12.

Google Drive (2021), "Hostel Image Dataset 7,350.zip", available at: https://drive.google.com/file/d/1byxXEIA1wvReSjlRW4k5Y1JFyuLnLrHy/view?usp=sharing (accessed 3 June 2021)

Greater London Authority (2020), "Mayor offers funding to make homeless hostels Covid19 safe", available at: https://www.london.gov.uk/press-releases/mayoral/mayor-offers-funding-to-make-hostels-covid-safe (accessed $\underline{3} 1$ JuneOctober 2021日)

He, K., Zhang, X., Ren, S. and Sun, J. (2015), "Deep residual learning for image recognition”, available at: https://arxiv.org/pdf/1512.03385.pdf (accessed 312 JuneOctober 2021 $\theta$ ) 
ImageNet (2020), "Large scale visual recognition challenge", available at: http://image-net.org/challenges/LSVRC/ (accessed $\underline{3} 1$ JuneOctober 20210)

Kanchinadam, T. (2016), "Hotel image classification using deep learning", available at: https://tkanchin.github.io/images/ml_report.pdf (accessed 12 October 2020)

Kaggle (2016), "Hotel image classification”, available at: https://www.kaggle.com/c/hotel-image-classification/overview(accessed $\underline{3} 12$ JuneOctober 20210)

Landola, F., Han, S., Moskewicz, M., Ashraf, K., Dally, W. And Keutzer, K. (2016), "SqueezeNet: AlexNet-level accuracy with 50x

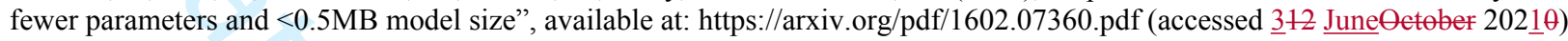

Li, F., Kant, S., Araki, S., Bangera, S. and Shukla, S. (2020), "Neural networks for fashion image classification and visual search", available at: https://arxiv.org/abs/2005.08170 (accessed 3 15 Junely 20210)

Mason, P. (2014), Researching tourism, leisure and hospitality for your dissertation, Goodfellow, Oxford, UK.

Mateen, M., Wen, J., Nasrullah, N., Sun, S. and Hayat, S. (2020), "Exudate detection for diabetic retinopathy using pretrained convolutional neural networks“, Complexity, Vol. 2020, pp.1-11.

MathWorks (20210), "Pretrained deep neural networks", available at:

https:/uk.mathworks.com/help/deeplearning/ug/pretrained-convolutional-neural-networks.html (accessed $\underline{3} 1$ JuneOctober 2021日)

O’Dea, S. (20210), "Number of smartphone users worldwide from 2016-202ㅌ1", available at:

https://www.statista.com/statistics/330695/number-of-smartphone-users-worldwide/ (accessed $\underline{3} 12$ June@etober 2021日)

OECD (2020), "Rebuilding tourism for the future: COVID-19 policy responses and recovery", available at:

http://www.oecd.org/coronavirus/policy-responses/rebuilding-tourism-for-the-future-covid-19-policy-responses-and-recoverybced9859/ (accessed 3 t

Premchaiswadi, W. et al, Tungkatsathan, A. and Premchaiswadi, N. (2010), "Mobile image search for tourist information using ACC algorithm", available at:

https://ieeexplore.ieee.org/abstract/document/5671757?casa_token=9U9V4B9AdmcAAAAA:NIkmA12MVs0anmxH-

RIMcrkAFti80mFyXILgf_NIXQMeN27De1vG31_cWDLfHCQ16XqMLg (accessed 31 June 2021 $\theta$ )

Raisi, Z., Mohanna, F. and Rezaei, M. et al. (2011), "Content-based image retrieval for tourism application”, available at:https://ieeexplore.ieee.org/abstract/document/6121857?casa_token=MF2x6Fvh3J4AAAAA:bjgdsbZ4jshw6PiK54QCHqQWK KsusDC7vAUG5cJ1ZTxUUAA5diCORIfknpwqLgd9WeRpFEM (accessed $\underline{3}+$ June 20210)

Raisi, Z., Mohanna, F. and Rezaei, M. et al. (2014), “Applying content-based image retrieval techniques to provide new service for tourism industry“, International journal advanced networking and applications, Vol. 6 No. 2, pp.2222-2232.

Rath, S. (2020), "Getting 95\% accuracy on the caltech101 dataset using deep learning", available at: https://debuggercafe.com/getting-95-accuracy-on-the-caltech101-dataset-using-deep-learning/ (accessed 31 June 2021 $\theta$ )

Rath, T. (2018), "Hotel image categorization with deep learning”, available at https://medium.com/kayak-tech/hotel-imagecategorization-with-deep-learning-ffa8429e55b5 (accessed 312 JuneOetober 2021 $\theta$ )

Ren, M., Vu, H., Li, G. and Law, R. (2020), "Large-scale comparative analyses of hotel photo content posted by managers and customers to review platforms based on deep learning: implications for hospitality marketers", available at https://www.tandfonline.com/doi/pdf/10.1080/19368623.2020.1765226?needAccess=true\& (accessed 312 JuneOctober 2021日)

Sabharwal, A. (2017), "500 million people using Google Photos and three new ways to share", available at: https://blog.google/products/photos/google-photos-500-million-new-sharing/(accessed $\underline{3}+\underline{\text { JuneMay } 2021 \theta)}$ 

communication and aerospace technology, IEE conference record, pp.656-660.

Stadlen, A. (2019), "Update on creative commons licenses and in memoriam accounts", available at: https://blog.flickr.net/2019/03/08/update-on-creative-commons-licenses-and-in-memoriam-accounts/ (accessed 1 May 2020)

Stylianou, A., Xuan, H., Shende, M., Brandt, J., Souvenir, R. and Pless, R. (2019), "Hotels-50K: a global hotel recognition dataset", available at: https://www.aaai.org/ojs/index.php/AAAI/article/view/3863 (accessed $\underline{3} 15$ Junely 2021 $\theta$ )

Szegedy, C., Liu, W., Jia, Y., Sermanet, P., Reed, S., Anguelov, D. and Rabinovich, A. (2015), "Going deeper with convolutions", paper presented at the IEEE conference on computer vision and pattern recognition, available at: https://www.cvfoundation.org/openaccess/content_cvpr_2015/html/Szegedy_Going_Deeper_With_2015_CVPR_paper.html (acce ssed 1 October 2020).

Tasli, E. (2017), “Automated image tagging at Booking.com”, available at: https://booking.ai/automated-image-tagging-at-bookingcom-7704f27dcc8b (accessed $\underline{3} 12$ JuneOctober 20210)

TankovskaClement, HJ. (20210), "Hours of video uploaded to YouTube every minute 2007-2019”, available at: https://www.statista.com/statistics/259477/hours-of-video-uploaded-to-youtube-every-minute/ (accessed 312 JuneOctober 2021日)

Wallace, G. K. (1991), “The JPEG still picture compression standard “, Communications of the ACM, Vol. 34 No. 4, pp.31-45.

Wengert, C., Douze, M. and Jegou, H. (2011), "Bag-of-colours for improved image search”, available at: https://dl.acm.org/doi/pdf/10.1145/2072298.2072034 (accessed $\underline{3} 1$ JuneMay 2021 0 )

Xiao, T., Liu, L., Li, K., Qin, W., Yu, S. and Li, Z. (2018), “Comparaison of transferred deep neural networks in ultrasonic breast masses discrimination“, Biomed research international, Vol. 2018, pp.1-9.

Zhao, B. Huang, B. and Zhong, Y. (2017), “Transfer learning with fully pretrained deep convolution networks for land-use classification “, IEEE geoscience and remote sensing letters, Vol. 14 No. 9, pp.1436-1440.

Zheng, L., Wang, S. and Tian, Q. (2014), “Coupled binary embedding for large-scale image retrieval “, IEEE transactions on image processing, Vol. 23 No. 8, pp.3368-3380.

Zhou, B., Lapedriza, A., Khosla, A., Oliva, A. and Torralba, A. (2017), "Places: a 10 million image database for scene recognition", working paper, Computer science and artificial intelligence laboratory, Massachusetts Institute of Technology, USA.

Zhu, L., Shen, J., Jin, H., Zheng, R. and Xie, L. (2015), “Content-based visual landmark search via multimodal hyper graph learning“, IEEE transactions on cybernetics, Vol. 45 No. 12, pp.2756-2769. 